\title{
Matching Logic: The Foundation of the K Framework (Invited Talk)
}

\author{
Grigore Roşu \\ University of Illinois at Urbana-Champaign \\ USA \\ Runtime Verification \\ USA \\ grosu@illinois.edu
}

\begin{abstract}
The $\mathrm{K}$ framework (kframework.org) is an effort in realizing the ideal language framework, where programming languages must have formal semantics and all language tools are automatically generated from the formal semantics. Until recently, $\mathrm{K}$ has been developed as an engineering endeavor driven by challenges such as formalizing the complete semantics of large languages (C, Java, JavaScript, Python, etc), but deriving its semantics from translations to various formalisms, such as rewriting logic, graph transformations, or Coq. This semantics borrowing approach came not only at a notational cost, where the original language meaning was "lost in translation", but also at a foundational cost: the target formalisms were more complicated than necessary, yet more restricted due to their prescribed ways to define language semantics.

In this talk I will present matching logic as the new and direct foundation of K. I will also discuss some of its applications in defining constructors, transition systems, modal mu-logic and temporal logic variants, reachability logic and thus Hoare logics, and separation-logic-style recursive predicates and patterns. Matching logic can therefore be regarded as an expressive foundation for programming languages, and $\mathrm{K}$ as a best effort implementation. An appealing aspect of matching logic's Hilbert-style proof system is that it admits a small proof checker, in the order of 200 LOC including parsing.
\end{abstract}

\author{
Xiaohong Chen \\ University of Illinois at Urbana-Champaign \\ USA \\ xc3@illinois.edu
}

CCS Concepts - Theory of computation $\rightarrow$ Logic; Semantics and reasoning; $\bullet$ Software and its engineering $\rightarrow$ Formal language definitions.

Keywords Language frameworks; formal semantics; logic ACM Reference Format:

Grigore Roşu and Xiaohong Chen. 2020. Matching Logic: The Foundation of the K Framework (Invited Talk). In Proceedings of the 9th ACM SIGPLAN International Conference on Certified Programs and Proofs (CPP '20), Fanuary 20-21, 2020, New Orleans, LA, USA. ACM, New York, NY, USA, 1 page. https://doi.org/10.1145/3372885. 3378574

Permission to make digital or hard copies of part or all of this work for personal or classroom use is granted without fee provided that copies are not made or distributed for profit or commercial advantage and that copies bear this notice and the full citation on the first page. Copyrights for thirdparty components of this work must be honored. For all other uses, contact the owner/author(s).

CPP '20, January 20-21, 2020, New Orleans, LA, USA

(C) 2020 Copyright held by the owner/author(s).

ACM ISBN 978-1-4503-7097-4/20/01.

https://doi.org/10.1145/3372885.3378574 